\title{
Giant myoma associated with uterine prolapse during the second trimester of twin pregnancy: case report
}

\begin{abstract}
A 38-years-old primigravida with twin dichorionic diamniotic pregnancy at 18 weeks of gestational age, had a giant myoma attached to the uterine fundus, resulting in uterine prolapse and restrictive gastric capacity. The patient went through a myomectomy at 18 weeks of gestational age. After the surgery, the patient was able to eat without vomiting and had a complete regression of uterine prolapse. One of the fetuses died two weeks after surgery, and the other was delivered by a C-section on the $363 / 7$ weeks. The postoperative period was uneventful, and the mother and newborn were discharged four days later.
\end{abstract}

Keywords: twin pregnancy, dichorionic diamniotic, giant myoma, uterine prolapse, myomectomy

\author{
Volume 12 Issue 4 - 202I
}

\section{Camila Poccetti Ribeiro,' Akemi Dafne \\ Dasha Koiti Nakamura,' Tamires de Menezes \\ França, ${ }^{2}$ Gustavo Rubino de Azevedo \\ Focchi, ${ }^{3}$ Gabriel Augusto Limone, ${ }^{3}$ Maria \\ Gabriela Baumgarten Kuster, ${ }^{2}$ Roney Cesar \\ Signorini Filho,' Rosiane Mattar,' Sue Yazaki \\ Sun'}

'Departamento de Obstetrícia, Escola Paulista de Medicina (EPM), Universidade Federal de São Paulo (UNIFESP), São Paulo, Brasil

${ }^{2}$ Departamento de Ginecologia, Escola Paulista de Medicina (EPM), Universidade Federal de São Paulo (UNIFESP), São Paulo, Brasil

${ }^{3}$ Departamento de Patologia, Escola Paulista de Medicina (EPM), Universidade Federal de São Paulo (UNIFESP), São Paulo, Brasil

\author{
Correspondence: Camila Poccetti Ribeiro, MD, Escola \\ Paulista de Medicina (EPM), Universidade Federal de São Paulo \\ (UNIFESP), Rua Machado Bittencourt, 300, ap 104, Zip Code \\ 04044-000 São Paulo, SP, Brasil,Tel +55 II 972843996, \\ Email camipoccetti@gmail.com
}

Received: July 18, 2021 | Published: July 27, 2021

\section{Introduction}

Uterine fibroids are frequently recorded benign tumors of the female reproductive system, affecting $20 \%-60 \%$ of women of reproductive age. ${ }^{1,2}$ In pregnant women, fibroids are known to occur in $0.1 \%-10.7 \%$, and the incidence increases as women postpone childbearing. ${ }^{3}$

Leiomyomas have been associated with increased frequency of spontaneous abortion, preterm labor, premature rupture of membranes, antepartum bleeding, placental abruption, anomalous fetal position, and cesarean section. ${ }^{4}$

There are few studies associating twin pregnancies, leiomyomas, and the outcome. Moreover, the incidence of twin pregnancies has increased in recent years, and the rate of fibroids in twin pregnancies has been estimated to be around $2.3 \%{ }^{5}$

The objective of this study is to report a case of a twin pregnancy and a giant myoma treated by myomectomy in the second trimester and the following prenatal care and delivery.

\section{Methods}

A primigravida, 38-year-old, black, was referred to our tertiary hospital on January 14th, 2020, presenting a dichorionic diamniotic twin pregnancy at $182 / 7$ weeks. She had a progressive eating difficulty initiated two months before the visit, vomiting practically all ingested food and liquids. Moreover, she presented an exacerbated increase of abdominal volume, decreased diuresis secondary to dehydration, and finally, a uterine prolapse.

On admission, blood pressure was $110 / 80 \mathrm{mmHg}$, pulse $110 \mathrm{bpm}$, $22 \mathrm{ipm}$ respiratory rate, $103.4 \mathrm{~kg}$ body weight, $36 \mathrm{~kg} / \mathrm{m}^{2}$ body mass index. The patient was also dehydrated. We observed an abdominal mass in the uterine fundus reaching the superior abdomen and the xiphoid process. Heartbeats of two fetuses were identified. An irreducible stage 3 uterine prolapse (POP-Q) was observed (Figure 1), and a moderated edema in the lower limbs.

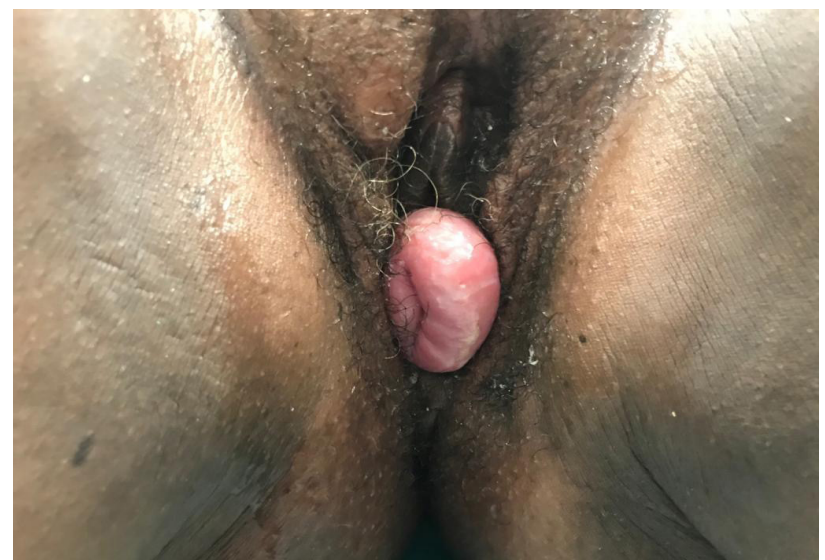

Figure I Irreducible stage 3 uterine prolapse (POP-Q): exteriorization of the uterine cervix from the vagina. 
On admission, her blood tests showed anemia (Table 1). Obstetric ultrasound revealed dichorionic diamniotic twin pregnancy, both fetuses alive and biometry compatible with menstrual age, corporal placenta, and normal amniotic fluid.

Table I Laboratory tests performed during twin pregnancy

\begin{tabular}{ll}
\hline Laboratory test & Results \\
\hline Hemoglobin $(|5 / 0| / 2020)$ & $8.1 / \mathrm{dL}$ \\
Hematocrit $(|5 / 0| / 2020)$ & $24.70 \%$ \\
Leukocytes(I5/0I/2020) & 13930 \\
Platelets (I5/0I/2020) & 327000 \\
Fasting blood glucose (I4/02/2020) & $89 \mathrm{mg} / \mathrm{dL}$ \\
Blood group (I5/0I/2020) & B \\
RhD Factor (I5/0I/2020) & Positive \\
HIV Serology (I5/0I/2020) & Non-reactive \\
Syphilis Serology (VDRL) & Non-reactive \\
(I5/0I/2020) & \\
Creatinine (I5/0I/2020) & $\begin{array}{l}\text { value: } 0.5 \text { to } 0.9 \mathrm{mg} / \mathrm{dL}) \\
\end{array}$ \\
Urea (I5/0I/2020) & $16 \mathrm{mg} / \mathrm{dL}$ (normal value: \\
\hline
\end{tabular}

Abdominal and pelvic magnetic resonance without contrast (Figure 2), showed increased uterine size, twin pregnancy, and a giant subserous myoma in the right side of the uterus, reaching the abdominal cavity. The mass measured about $30.0 \times 24.0 \mathrm{~cm}$, compressing the ureters bilaterally and accentuated collector systems' dilation. Also, there were cystic and bleeding areas (red degeneration).

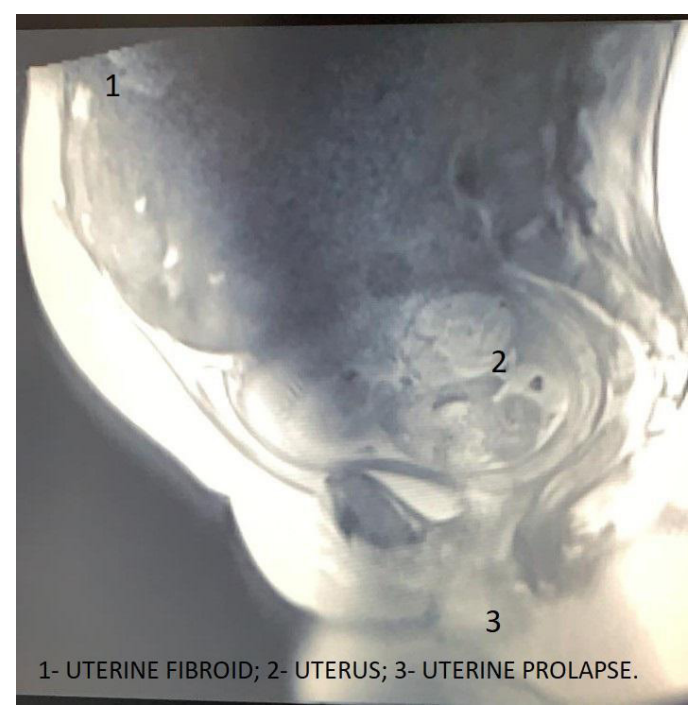

Figure 2 Magnetic Resonance image. Sagittal Section.

The patient was subjected to a myomectomy based on the clinical condition and the exams. The incision was infra- and supra-umbilical midline and longitudinal, under spinal and general anesthesia. In the abdominal cavity, we observed an umbilical hernia with adhesions from the omentum to the intestinal mucosa with no sign of intestinal loops or strangulation inside. Cavity inspection detected a citrus yellow liquid located in the parieto-colic gutter, forwarded for pathological study.

Furthermore, a large mass measuring around $35 \times 25 \times 20 \mathrm{~cm}$ was observed, occupying the entire abdominal upper floor, with multiple adhesions in the supra and infra mesocolic omentum located in its anterior wall. We performed an infracolic partial omentectomy, releasing the stomach, which was compressed and closely attached to this mass by the minor omentum. Uterine visualization was possible, confirming the fibroid process. The giant myoma had a pedicle measuring approximately $5 \mathrm{~cm}$ in length and $10 \mathrm{~cm}$ in width, located in the fundus's anterior part. The uterus was near the umbilical scar, and it was being pushed against the pelvis by the myoma.

After the exeresis of the pedunculated myoma (Figures $3 \mathrm{~A}$ ) weighing $6,355 \mathrm{~g}$ (Figure 3B), and measuring about $36.5 \times 30.0 \times 12.0 \mathrm{~cm}$, the bloody area from the myomectomy was sutured with separated stitches, achieving an excellent hemostatic control. Blood transfusion was not necessary (hemoglobin $-9.7 \mathrm{~g} / \mathrm{dl}$ and hematocrit $-29.8 \%$ postoperative). The patient was discharged on the fourth postoperative day with her newborn alive, showing complete regression of the uterine prolapse the following day after the surgery (Figure 3B).

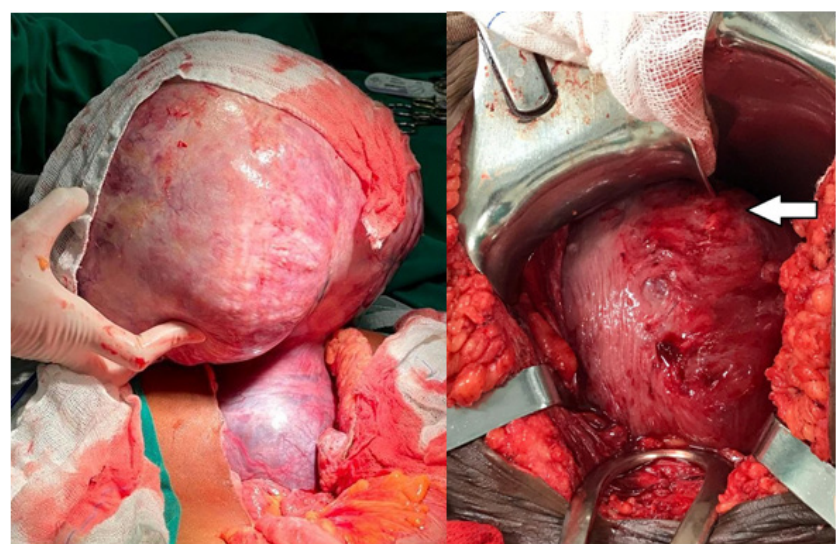

Figure 3 A. Pedunculated myoma in the uterine wall. B. Intraoperative appearance after uterine repair.The arrow highlights the scar region from the previous myomectomy performed at about 18 weeks of gestation.

The anatomopathological study showed a globular tumour with a smooth and regular external surface. Inside the tumor was observed a homogeneous surface, tangled appearance, whitish color, firm consistency and areas of cystification. Regarding the histological examination, we observed an architecturally organized neoplasm in long orthogonal bundles, presenting cells without atypia, without mitotic activity, and differentiated smooth muscle phenotype. This structure is compatible with leiomyoma. There were areas of necrosis and pseudocystification, also common to leiomyomas.

\section{Postoperative follow- up}

On January 30, 2020, we verified one fetus's death through an obstetric ultrasound on the fourteenth postoperative day. Despite the death of one of the twins, the gestation followed with no complications until 36 weeks and 3 days, when it was interrupted due to fetal vitality compromise.

During abdominal cavity opening, adhesions between the uterus, small bowel loops, and an intramural myoma- about $5 \mathrm{~cm}$ in the body region - were undone. We performed a transverse segmental hysterotomy with a large amount of clear amniotic fluid and a cephalic 
extraction of the fetus, which had two tightened circular cords in the cervical region. The newborn weighed $2180 \mathrm{~g}$, Apgar score of 7 in the first minute and 9 in the fifth minute. Still, an intrapartum insertion of copper IUDs was performed, complication-free.

The postpartum period was uneventful, and she was discharged on the second postoperative day, together with low molecular weight heparin use for 40 days after delivery.

The anatomopathological analysis of the placenta showed PLACENTA (1): weight 430g -Chorionic villi compatible with the $3^{\text {rd }}$ gestational quarter showing areas of infarction; -Absence of histological changes in the membranes and umbilical cord. PLACENTA (2) weight $180 \mathrm{~g}$ - Dichorionic and diamniotic placenta with fetal death; - Diffuse tissue autolysis in the membranes and umbilical cord.

On the 40th postpartum day, the patient had no complaints. The ultrasound showed a properly positioned intrauterine device. The newborn was growing and developing well.

\section{Discussion}

There are inconsistent data on the impact of myoma on pregnancy and even less data regarding how they influence twin pregnancies' obstetric outcomes. It has been reported that around $10 \%$ of pregnant women with myomas suffer complications related to the presence of these tumors. ${ }^{3}$ Such complications include spontaneous abortion, antepartum bleeding, premature rupture of membranes, preterm labor, placenta previa, postpartum bleeding, and a high incidence of cesarean deliveries. ${ }^{3,6-8}$ To the best of our knowledge, this is the first report of prolapse uterine caused by giant myoma during a twin pregnancy.

Indications for myomectomy during pregnancy include severe abdominal pain due to torsion of subserosal pedunculated myomas, red degeneration unresponsive to medical treatment, and an increase in myoma's size, leading to abdominal discomfort. It has been reported that if symptoms persist after 72 hours of therapy, then surgical intervention must be considered. ${ }^{3}$

The case reported here demonstrated a successful approach to a twin pregnancy on a patient with giant myoma, resulting in critical consequences to the mother's quality of life, including difficulties to eat. Therefore, compromising fetal development. Moreover, the significant uterine prolapse seen here and the associated gastric symptoms suggested that the surgical intervention was the unique possible treatment.

The death of one of the twins was diagnosed fourteen days later in a routine exam. We assume that the cause of the death was the myoma's placental blood vessels since the fetus' placenta was inserted in the anterior body wall near the mass.

After, the C-section could be performed on an elective day, but due to fetal distress detected in cardiotocography, the delivery was anticipated. Despite that, it was an uneventful C-section, resulting in good recovery with a late preterm delivery, with no complications. Thanks to the medical team working together, a rare case not reported in the literature was successfully resolved.

\section{Conclusion}

Surgical management of myomas during pregnancy may be performed successfully in carefully selected patients, resulting in successful pregnancy outcomes.

\section{Acknowledgments}

None.

\section{Funding}

None.

\section{Ethics committee}

Approval number: 35896920.2.0000.5505.

\section{Conflicts of interest}

The authors declare that they have no conflicts of interest.

\section{References}

1. Lai J, Caughey AB, Qidwai GI, et al. Neonatal outcomes in women with sonographically identified uterine leiomyomata. J Matern Neonatal Med. 2012;25(6):710-713.

2. Wang HM, Tian YC, Xue ZF, et al. Associations between uterine fibroids and obstetric outcomes in twin pregnancies. Int $J$ Gynecol Obstet. 2016;135(1):22-27

3. Lolis DE, Kalantaridou SN, Makrydimas G, et al. Successful myomectomy during pregnancy. Hum Reprod. 2003;18(8):1699-1702.

4. Stout MJ, Odibo AO, Shanks AL, et al. Fibroid tumors are not a risk factor for adverse outcomes in twin pregnancies. Am J Obstet Gynecol. 2013;208(1):68.e1-68.e5

5. Rice JP, Kay HH, Mahony BS. The clinical significance of uterine leiomyomas in pregnancy. Am J Obstet Gynecol. 1989;160(5 PART 1):1212-1216.

6. Vergani P, Ghidini A, Strobelt N, et al. Do uterine leiomyomas influence pregnancy outcome? Obstet Gynecol Surv. 1995;50(3):165-6.

7. Benson CB, Chow JS, Chang-Lee W, et al. Outcome of pregnancies in women with uterine leiomyomas identified by sonography in the first trimester. J Clin Ultrasound. 2001;29(5):261-264.

8. Dildy GA, Moise KJ, Smith LG, et al. Indomethacin for the treatmen of symptomatic leiomyoma uteri during pregnancy. Obstet Gynecol Surv. 1993;48(1):15-17. 